\title{
Warm water deuterium fractionation in IRAS 16293-2422 ${ }^{\star}$ The high-resolution ALMA and SMA view
}

\author{
M. V. Persson ${ }^{1,2}$, J. K. Jørgensen ${ }^{2,1}$, and E. F. van Dishoeck ${ }^{3,4}$ \\ 1 Centre for Star and Planet Formation, Natural History Museum of Denmark, University of Copenhagen, Øster Voldgade 5-7, \\ 1350 Copenhagen K, Denmark \\ e-mail: magnusp@nbi.dk \\ 2 Niels Bohr Institute, University of Copenhagen, Juliane Maries Vej 30, 2100 Copenhagen Ø, Denmark \\ 3 Leiden Observatory, Leiden University, PO Box 9513, 2300 RA Leiden, The Netherlands \\ ${ }^{4}$ Max-Planck Institute für extraterrestrische Physik (MPE), Giessenbachstrasse, 85748 Garching, Germany \\ Received 26 October 2012 / Accepted 28 November 2012
}

\section{ABSTRACT}

\begin{abstract}
Context. Measuring the water deuterium fractionation in the inner warm regions of low-mass protostars has so far been hampered by poor angular resolution obtainable with single-dish ground- and space-based telescopes. Observations of water isotopologues using (sub)millimeter wavelength interferometers have the potential to shed light on this matter.

Aims. To measure the water deuterium fractionation in the warm gas of the deeply-embedded protostellar binary IRAS 16293-2422. Methods. Observations toward IRAS 16293-2422 of the $5_{3,2}-4_{4,1}$ transition of $\mathrm{H}_{2}^{18} \mathrm{O}$ at $692.07914 \mathrm{GHz}$ from Atacama Large Millimeter/submillimeter Array (ALMA) as well as the $3_{1,3}-2_{2,0}$ of $\mathrm{H}_{2}^{18} \mathrm{O}$ at $203.40752 \mathrm{GHz}$ and the $3_{1,2}-2_{2,1}$ transition of HDO at $225.89672 \mathrm{GHz}$ from the Submillimeter Array (SMA) are presented.

Results. The $692 \mathrm{GHz} \mathrm{H}{ }_{2}^{18} \mathrm{O}$ line is seen toward both components of the binary protostar. Toward one of the components, "source B", the line is seen in absorption toward the continuum, slightly red-shifted from the systemic velocity, whereas emission is seen off-source at the systemic velocity. Toward the other component, "source A", the two $\mathrm{HDO}$ and $\mathrm{H}_{2}^{18} \mathrm{O}$ lines are detected as well with the SMA. From the $\mathrm{H}_{2}^{18} \mathrm{O}$ transitions the excitation temperature is estimated at $124 \pm 12 \mathrm{~K}$. The calculated $\mathrm{HDO} / \mathrm{H}_{2} \mathrm{O}$ ratio is $(9.2 \pm 2.6) \times 10^{-4}-$ significantly lower than previous estimates in the warm gas close to the source. It is also lower by a factor of $\sim 5$ than the ratio deduced in the outer envelope.

Conclusions. Our observations reveal the physical and chemical structure of water vapor close to the protostars on solar-system scales. The red-shifted absorption detected toward source B is indicative of infall. The excitation temperature is consistent with the picture of water ice evaporation close to the protostar. The low $\mathrm{HDO} / \mathrm{H}_{2} \mathrm{O}$ ratio deduced here suggests that the differences between the inner regions of the protostars and the Earth's oceans and comets are smaller than previously thought.
\end{abstract}

Key words. astrochemistry - stars: formation - protoplanetary disks - ISM: abundances - ISM: general

\section{Introduction}

Water plays an essential role for life as we know it, but its origin on Earth is still unclear: was water accreted during the early stages of Earth's formation, or brought by smaller solar system bodies such as comets at later times? To deduce the origin of Earth's water and the amount of chemical processing it has experienced, one option is to measure the water deuterium fractionation $\left(\mathrm{HDO} / \mathrm{H}_{2} \mathrm{O}\right)$ during different stages in the evolution of protostars and compare it to what we measure in Earth's oceans and comets.

Generally the $\mathrm{HDO} / \mathrm{H}_{2} \mathrm{O}$ ratio in Earth's oceans of $3 \times 10^{-4}$ (e.g., Lécuyer et al. 1998) and Oort cloud comets of $8.2 \times 10^{-4}$ (Villanueva et al. 2009) are found to be enhanced above the $\mathrm{D} / \mathrm{H}^{1}$ ratio in the protosolar nebula $\sim 1.5 \times 10^{-5}$ (Linsky 2003 ; Geiss \& Gloeckler 1998) due to deuterium fractionation processes. The factor of 2 higher abundance ratio in the Oort cloud comets than in Earth's oceans has previously been taken as an indication that only a small fraction of Earth's water was delivered by comets. Recently however, a $\mathrm{HDO} / \mathrm{H}_{2} \mathrm{O}$ ratio of $3.2 \times 10^{-4}$ was measured

* Appendix A is available in electronic form at http: //www . aanda. org

$1 \mathrm{~A} \mathrm{HDO} / \mathrm{H}_{2} \mathrm{O}$ ratio of " $x$ " corresponds to a $\mathrm{D} / \mathrm{H}$ ratio of " $x / 2$ ". for the Jupiter class comet Hartley 2 with the Herschel Space Observatory (Hartogh et al. 2011) and $4.2 \times 10^{-4}$ for the Oort cloud comet Garradd (Bockelée-Morvan et al. 2012), indicating values closer to those of Earth's water.

Attempts at measuring the water deuterium fractionation in protostars have resulted in different conclusions. Parise et al. (2003) used ground-based infrared observations of the stretching bands of $\mathrm{OH}$ and $\mathrm{OD}$ in water ice in the outer parts of envelopes and found upper limits ranging from $0.5 \%$ to $2 \%$ for the $\mathrm{HDO} / \mathrm{H}_{2} \mathrm{O}$ ratios in four embedded low-mass protostars. In the gas-phase it is possible to detect lines of HDO, but such studies differ on the interpretation with $\mathrm{HDO} / \mathrm{H}_{2} \mathrm{O}$ ratios in protostars ranging from cometary values (Stark et al. 2004), to a few \% (Parise et al. 2005; Liu et al. 2011). Even more recently, Coutens et al. (2012) deduced a $\mathrm{HDO} / \mathrm{H}_{2} \mathrm{O}$ ratio in IRAS $16293-2422$ of $3.4 \times 10^{-2}$ in the inner parts and $0.5 \times 10^{-2}$ in the outer envelope by modeling a large range of lines observed with Herschel.

One problem with previous measurements of $\mathrm{HDO} / \mathrm{H}_{2} \mathrm{O}$ is the relatively large beam size of single-dish ground- and spacebased telescopes. Spherically symmetric power-law models of protostellar envelopes have usually been employed to interpret the observations. While such models are appropriate to interpret continuum and line emission on larger scales (>300 AU), 
they are not suited to unambiguously analyze the observed compact components since there are clear indications that they are not an accurate representation of the conditions on small scales (e.g., Jørgensen et al. 2005; Chiang et al. 2012). Estimates of abundance ratios on these smaller scales are thus subject to significant uncertainties due to extrapolations of the underlying physical structures.

High angular resolution millimeter wavelength aperture synthesis observations offer a possibility to circumvent this issue. Recently Jørgensen \& van Dishoeck (2010) detected the water isotopologue $\mathrm{H}_{2}^{18} \mathrm{O}$ toward the deeply embedded protostar NGC-1333 IRAS4B on scales of $<50$ AU using the IRAM Plateau de Bure Interferometer (PdBI), which combined with an upper limit on the HDO column density from the SMA resulted in a $3 \sigma$ upper limit to the $\mathrm{HDO} / \mathrm{H}_{2} \mathrm{O}$ abundance ratio of $6 \times 10^{-4}$. To follow-up these results we initiated an extended survey of the $\mathrm{H}_{2}^{18} \mathrm{O}$ and HDO emission on arcsecond scales using the IRAM PdBI and SMA (Persson et al. 2012).

IRAS $16293-2422$ is a Class 0 protostellar binary (sep $\sim 5^{\prime \prime}$, 600 AU) located $120 \mathrm{pc}$ away in the LDN $1689 \mathrm{~N}$ cloud in the $\rho$ Ophiucus star-forming region (Knude \& Høg 1998; Loinard et al. 2008). With a rich spectrum at (sub)millimeter wavelengths (Blake et al. 1994; van Dishoeck et al. 1995; Cazaux et al. 2003; Chandler et al. 2005; Caux et al. 2011; Jørgensen et al. 2011) it has been one of the prime targets for studies of astrochemistry during the star-formation process, revealing the presence of a range of complex organic species (Bottinelli et al. 2004; Kuan et al. 2004; Bisschop et al. 2008) and prebiotic molecules (Jørgensen et al. 2012) on (sub)arcsecond scales.

In this letter we present, for the first time, high-resolution ground-based observations of several isotopologues of water toward IRAS 16293-2422 with both the ALMA ( 0.'2; 24 AU) and the SMA $(\sim 2.3 ; 276$ AU). We derive direct, modelindependent estimates of the water excitation temperature and use it to calculate the column density and the $\mathrm{HDO} / \mathrm{H}_{2} \mathrm{O}$ ratio in the warm inner envelope.

\section{Observations}

Observations of the deeply-embedded low-mass protostellar binary IRAS 16293-2422 were carried out at $690 \mathrm{GHz}$ with ALMA and $230 \mathrm{GHz}$ with the SMA, targeting the $5_{3,2}-4_{4,1}$ $(692.07914 \mathrm{GHz})$ and $3_{1,3}-2_{2,0}(203.40752 \mathrm{GHz})$ transitions of $\mathrm{H}_{2}^{18} \mathrm{O}$ and the $3_{1,2}-2_{2,1}(225.89672 \mathrm{GHz}$ ) transition of HDO (see Table A.1 in the Appendix). In the tables, the $203 \mathrm{GHz}$ observations are indicated with " 1 ", $225 \mathrm{GHz}$ observations with " 2 " and 692 GHz with "3" (see Tables 1 and A.1)

The ALMA observations of IRAS 16293-2422 were conducted as part of the ALMA Science Verification (SV) program: IRAS 16293-2422 was observed with 13 antennas on April 16 and 17, 2012 in a seven pointing mosaic centered at $\alpha=16 \mathrm{~h} 32 \mathrm{~m} 22^{\mathrm{s}} .7, \delta=-24^{\circ} 28^{\prime} 32^{\prime \prime} .5$ (J2000). The observations cover projected baselines from 26 to $402 \mathrm{~m}$ (62 to $945 \mathrm{k} \lambda$ ). One of the basebands was centered at $691.299 \mathrm{GHz}$ with a bandwidth of $1.875 \mathrm{GHz}$ and a spectral resolution of $0.923 \mathrm{MHz}$ $\left(0.4 \mathrm{~km} \mathrm{~s}^{-1}\right)$, a setup that covers the $\mathrm{H}_{2}^{18} \mathrm{O} \mathrm{53,2}-4_{4,1}$ line at 692.07914 GHz. Calibration observations include the quasars 1924-292 and 3c279 for the bandpass, the asteroid Juno for the amplitude, and the quasars 1625-254 and nrao530 for the phase. The science verification data are available as calibrated $u v$-data sets, which were used in our analysis. The calibrated data were imaged using the CASA software package (McMullin et al. 2007).
The lower-lying excited $\mathrm{H}_{2}^{18} \mathrm{O} \quad 3_{1,3}-2_{2,0}$ transition at 203.4075 GHz was observed with the SMA on May 1, 2011 in the compact configuration with seven antennas. This configuration resulted in projected baselines between 9 and $69 \mathrm{~m}$ (6 to $47 \mathrm{k} \lambda$ ). The passband was calibrated by observations of the quasar 3c279 while the absolute flux and complex gains were calibrated by observing Titan and the quasars 3c279, 1517-243 and 1626-298. The raw data calibration followed the standard recipes using the MIR package (Qi 2008) and then MIRIAD (Sault et al. 1995) was used to subtract the continuum from the data to create continuum-free line maps.

Finally, we utilized SMA observations of the HDO $3_{1,2}-2_{2,1}$ line at $225.89672 \mathrm{GHz}$ from the SMA (Jørgensen et al. 2011): those observations have a spectral resolution of $0.41 \mathrm{MHz}\left(0.54 \mathrm{~km} \mathrm{~s}^{-1}\right)$ and cover the projected baselines between 8.6-119.6 m (6.5-90 k $\lambda)$. For further information about those observations we refer to Jørgensen et al. (2011). The resulting beam size, field of view, velocity resolution and RMS of the various line data are summarized in Table A.1 in the Appendix.

\section{Results}

Figure 1 shows the spectra around the water lines toward the continuum peaks with Gaussian fits. Source A is in itself a binary (Chandler et al. 2005) and we here refer to the components as A1 (the northeast component) and A2 (the southwest component) and the lower resolution single component as A. Both $\mathrm{H}_{2}^{18} \mathrm{O}$ lines are clearly detected toward source $\mathrm{A}$ in emission, while only the $692 \mathrm{GHz} \mathrm{H}{ }_{2}^{18} \mathrm{O}$ line is seen toward source $\mathrm{B}$ in absorption. The $\mathrm{H}_{2}^{18} \mathrm{O}$ emission is clearly associated with peak A1 in the $692 \mathrm{GHz}$ data. The $\mathrm{H}_{2}^{18} \mathrm{O} 692 \mathrm{GHz}$ absorption line toward the continuum peak of source $\mathrm{B}$ is narrow and marginally red-shifted; the absorption is not due to the broad outflow seen in some lines (Jørgensen et al. 2011).

This weak absorption feature is not seen in the SMA data but the high sensitivity of ALMA combined with the stronger continuum at high frequencies is enough to detect it. The lines were identified using the JPL and CDMS catalogs through the Splatalogue compilation (Pickett et al. 1998; Müller et al. 2001).

The Splatalogue compilation shows no other possible line candidates at least $\pm 1 \mathrm{~km} \mathrm{~s}^{-1}$ from the line peak positions of the $\mathrm{H}_{2}^{18} \mathrm{O}$ line at $203 \mathrm{GHz}$ and the HDO line at $225 \mathrm{GHz}$. Lines that fall within $\pm 2-3 \mathrm{~km} \mathrm{~s}^{-1}$ exist, but are of too low intrinsic line strengths or lack additional components that should have been detected at other velocity offsets e.g., $\left(\mathrm{CH}_{3}\right)_{2} \mathrm{CO}$, ${ }^{13} \mathrm{CH}_{3} \mathrm{CH}_{2} \mathrm{CN}$ or $\mathrm{C}_{6} \mathrm{H}$. At 203.403 and $203.410 \mathrm{GHz}$ (i.e., about 10 and $-3 \mathrm{~km} \mathrm{~s}^{-1}$ ) lies $\mathrm{CH}_{3} \mathrm{OCH}_{3} 3_{3,1}-2_{2,1} \mathrm{EA} / \mathrm{EE}$, which is accounted for when fitting the $203 \mathrm{GHz}$ line. These lines are narrow enough to avoid any significant interference with $\mathrm{H}_{2}^{18} \mathrm{O}$ (see top most plot in Fig. 1).

For the $\mathrm{H}_{2}^{18} \mathrm{O}$ ALMA data at $692 \mathrm{GHz}$ the line identification in source $\mathrm{A}$ is complicated by the fact that the source is resolved and shows a systematic velocity pattern in other lines (Pineda et al. 2012). The line toward the continuum position of source A is red-shifted by about $2 \mathrm{~km} \mathrm{~s}^{-1}$ from the systemic $v_{\mathrm{LSR}}$ of $3.2 \mathrm{~km} \mathrm{~s}^{-1}$, similar to lines from other species (see Fig. 1, panel four). The third spectrum from the top in Fig. 1 shows the spectrum after smoothing with a Gaussian kernel of $1^{\prime \prime}$ size. The Gaussian fit to these data peaks closer to $v_{\mathrm{LSR}}=3.2 \mathrm{~km} \mathrm{~s}^{-1}$, indicative indeed of resolved velocity structure. Outflow emission (cf., Loinard et al. 2012), as also seen in $\mathrm{H}_{2} \mathrm{O}$ masers on small 


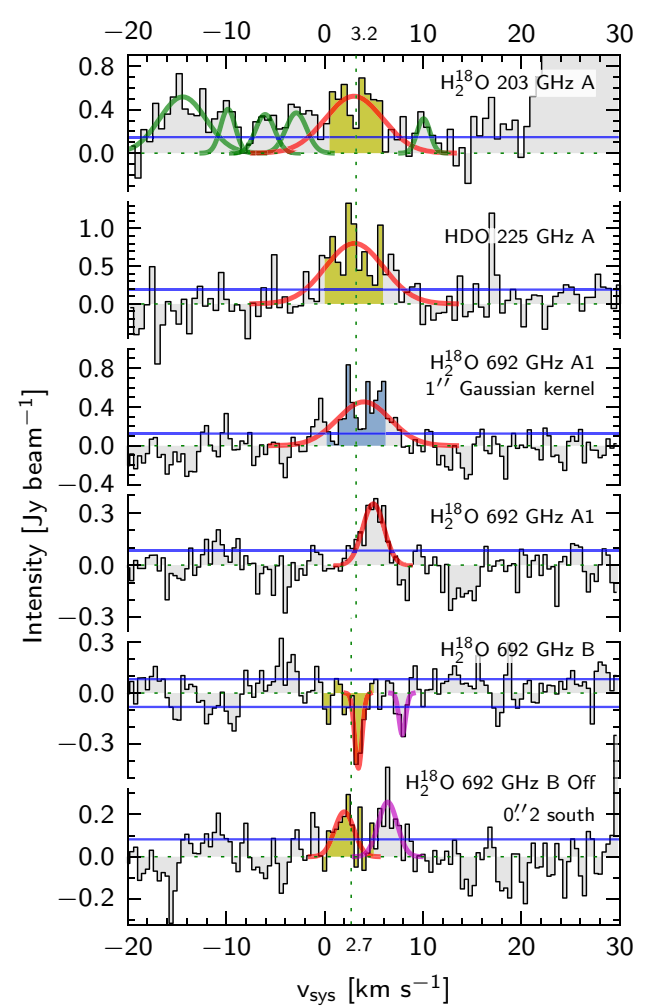

Fig. 1. Spectra of the targeted water lines toward both IRAS 16293$2422 \mathrm{~A}$ and B. The $203 \mathrm{GHz} \mathrm{H}{ }_{2}^{18} \mathrm{O}$ line spectrum (top) was binned to twice the resolution for clarity. The $1 \mathrm{D}$ Gaussian fits (red: $\mathrm{H}_{2}^{18} \mathrm{O}$; green: $\mathrm{CH}_{3} \mathrm{OCH}_{3}$; magenta: unidentified) and the RMS (blue) are plotted. Shaded areas show the interval over which the integrated intensities have been calculated (yellow and blue). The dotted green vertical lines shows $v_{\mathrm{LSR}}=3.2$ and $2.7 \mathrm{~km} \mathrm{~s}^{-1}$ for source $\mathrm{A}$ and $\mathrm{B}$ respectively. The third spectrum from the top (blue fill) is from data smoothed with a 1 " Gaussian kernel.

scales (Alves et al. 2012), could explain this velocity shift, but it is not possible to deduce its importance from the current data.

However, another possibility is that line blending causes the shift. Toward source B, the off position spectrum shows two emission lines, the second line at $\sim 6 \mathrm{~km} \mathrm{~s}^{-1}$ is unidentified (bottom spectrum Fig. 1). The $\mathrm{H}_{2}^{18} \mathrm{O}$ line at $692 \mathrm{GHz}$ toward source A may therefore also be affected by line blending. However, the unambiguous detection of the $692 \mathrm{GHz}$ line toward source B and the SMA observations of water toward source A suggest that at least half of the $692 \mathrm{GHz}$ feature toward source A is indeed due to the $\mathrm{H}_{2}^{18} \mathrm{O}$ line.

Table 1 lists parameters from Gaussian fits to the image and $u v$-plane of the line emission. The integrated intensity maps for the detected water lines are shown in Fig. 2.

\section{Discussion}

\subsection{Spectra and integrated intensity}

The different water lines toward source A show similar characteristics. The peak positions and line widths of the Gaussian fits to the $203 \mathrm{GHz} \mathrm{H} \mathrm{H}_{2}^{18} \mathrm{O}$ and $225 \mathrm{GHz}$ HDO lines are similar and agree with previous studies of other molecules toward IRAS 16293-2422 e.g., Bisschop et al. (2008); Jørgensen et al. (2011); Jørgensen et al. (2012); Pineda et al. (2012) (see Table 1). The mapped emission is compact and traces the warm water on scales $R<200$ AU.

Toward source $\mathrm{B}$, the $692 \mathrm{GHz} \mathrm{H}{ }_{2}^{18} \mathrm{O}$ line is detected in absorption. The spectral line is slightly red-shifted compared with $v_{\text {LSR }}=2.7 \mathrm{~km} \mathrm{~s}^{-1}$ of source $\mathrm{B}$, consistent with the picture of
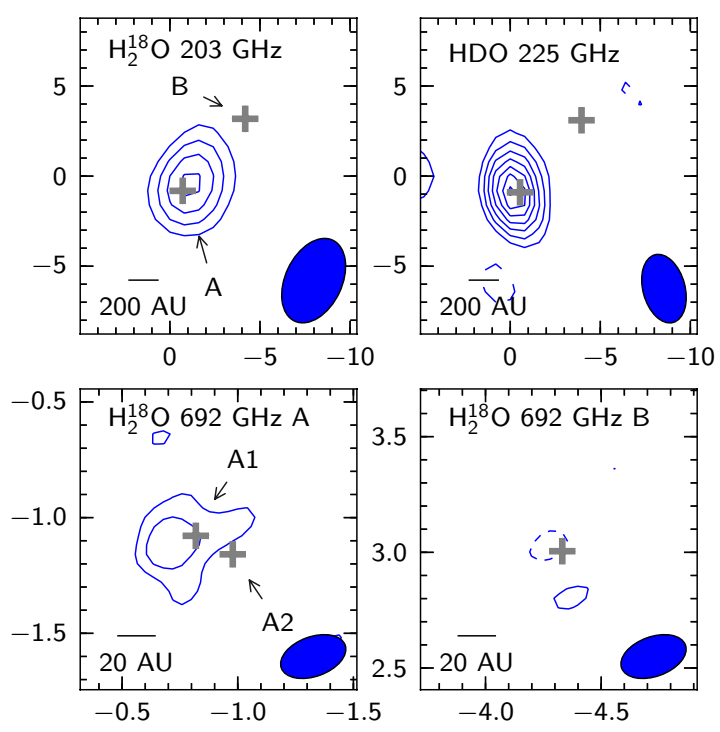

Fig. 2. Integrated intensity maps for all observed water lines calculated from channels \pm 3 from the systemic velocity, deduced from previous observations (Jørgensen et al. 2011). The absorption line toward source $\mathrm{B}$ was integrated in the interval $0-5 \mathrm{~km} \mathrm{~s}^{-1}$. Note the different spatial scales of the top vs the bottom panels. The beam is shown in the lower right corner and the gray crosses show the position of continuum peaks from elliptical Gaussian fits. Units on the axes are offset in arcseconds from the phase center of the $203 \mathrm{GHz}$ observations. Contours are in steps of $2 \sigma$ starting at $3 \sigma$, dashed contours represent negative values.

Table 1. Parameters from fits to the integrated maps and spectra of the different water lines for source A and B.

\begin{tabular}{llcl}
\hline \hline $\begin{array}{l}\text { Line } \\
\text { Id }\end{array}$ & $\begin{array}{l}\text { Size } \\
{\left[{ }^{\prime}\right]}\end{array}$ & $\begin{array}{l}\text { Intensity } \\
{\left[\mathrm{Jy} \mathrm{km} \mathrm{s}{ }^{-1}\right]}\end{array}$ & $\begin{array}{l}\text { Line width } \\
{\left[\mathrm{km} \mathrm{s}^{-1}\right]}\end{array}$ \\
\hline 1 (A) & $1.7 \pm 0.7$ & $3.9 \pm 1.4$ & $6.9 \pm 1.5$ \\
2 (A) & $1.1 \pm 0.4$ & $8.9 \pm 1.8$ & $7.0 \pm 0.7$ \\
3 (A1) & $0.2 \times 0.4\left(13^{\circ}\right)$ & $3.4 \pm 0.6$ & $6.3 \pm 0.7$ \\
3 (B) & Point fit & $-0.43 \pm 0.07$ & $0.9 \pm 0.2$ \\
\hline
\end{tabular}

Notes. The given errors are the statistical uncertainties and the $20 \%$ calibration uncertainty in intensity is not included. The spectral line fits were done toward the continuum peak and integrated intensities deduced from circular Gaussian fits in the $u v$-plane (203 and $225 \mathrm{GHz}$ ) or an elliptical Gaussian fit in image plane (692 GHz). The column "Line Id" gives the source indicated in parentheses, and the number corresponds to one of the lines (see text and Table A.1). The line width is the FWHM from a Gaussian fit to the spectral line. The elliptical Gaussian parameters (for line 3 (A1)) are given as minor and major axis $\left( \pm 00^{\prime} 1\right)$ and position angle $\left(\mathrm{PA}, \pm 4^{\circ}\right)$.

ongoing infall in this source (Jørgensen et al. 2012; Pineda et al. 2012). Integrating over a larger velocity interval reveals, in addition to the absorption, a blue-shifted emission peak, offset $\sim 0.2$ south of the absorption (see Figs. 1 and 2).

\subsection{Temperature and deuteration of water vapor}

To calculate the excitation temperature and column densities toward source A we assume LTE conditions and that the emission at 203 and $225 \mathrm{GHz}$ has the same extent as in the $692 \mathrm{GHz}$ observations (see Table 1). Scaling the $203 \mathrm{GHz} \mathrm{H}{ }_{2}^{18} \mathrm{O}$ intensities to the $692 \mathrm{GHz}$ source size gives an excitation temperature of $T_{\mathrm{ex}}=124 \pm 12 \mathrm{~K}$ for $\mathrm{H}_{2}^{18} \mathrm{O}$. If the $692 \mathrm{GHz}$ line is indeed blended, it would imply that the intensity estimate, and also the 
excitation temperature, are upper limits. Halving the intensity of the $692 \mathrm{GHz}$ line causes a drop to $T_{\mathrm{ex}}=107 \pm 9 \mathrm{~K}$. The extent and peak location of the emission in source A show that the warm water vapor is located at a small projected distance from the center of the protostar. The determined excitation temperature indicates that the distance is also small along the line of sight.

The estimated HDO column density is $4.8 \times 10^{17} \mathrm{~cm}^{-2}$, corrected for beam dilution when assuming that the extent of the

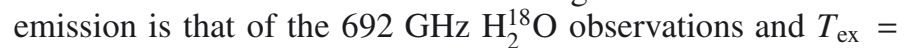
$124 \mathrm{~K}$. The gas-phase $\mathrm{H}_{2} \mathrm{O}$ column density is $5.3 \times 10^{20} \mathrm{~cm}^{-2}$ assuming the same as for HDO and that the isotopic abundance ratio of ${ }^{16} \mathrm{O} /{ }^{18} \mathrm{O}$ is 560 in the local interstellar medium (Wilson $\&$ Rood 1994). Assuming an uncertainty of about $20 \%$ for the column densities, originating in the flux calibration, the best estimate of the $\mathrm{HDO} / \mathrm{H}_{2} \mathrm{O}$ ratio is $(9.2 \pm 2.6) \times 10^{-4}$.

Given the uncertainty in the determination of the excitation temperature, testing the effect of different temperatures is important. If the excitation temperature is as low as $80 \mathrm{~K}$ the $\mathrm{HDO} / \mathrm{H}_{2} \mathrm{O}$ ratio becomes $7.8 \pm 2.2 \times 10^{-4}$. Increasing the excitation temperature to $300 \mathrm{~K}$ (Jørgensen et al. 2012) increases the ratio to $1.1 \pm 0.3 \times 10^{-3}$. The conclusions in this paper do not change over this wide interval in $T_{\mathrm{ex}}$. In addition, since the $203 \mathrm{GHz} \mathrm{H}_{2}^{18} \mathrm{O}$ and $225 \mathrm{GHz} \mathrm{HDO}$ observations have comparable beam and sources size, and arise from levels with similar energies, the deduced $\mathrm{HDO} / \mathrm{H}_{2} \mathrm{O}$ ratio is robust. However, if the HDO emission were more extended, its column density and the $\mathrm{HDO} / \mathrm{H}_{2} \mathrm{O}$ ratio would be lower. In this scenario our inferred ratio is an upper limit.

In contrast with previous estimates based on models of single-dish observations (Parise et al. 2005; Coutens et al. 2012), the deduced $\mathrm{HDO} / \mathrm{H}_{2} \mathrm{O}$ ratio in the warm gas of IRAS 162932422 is only slightly higher than found in Earth's oceans and by recent Herschel observations of comets. Given the possible systematic errors due to assumptions of the extent, they could be even closer. Within the statistical uncertainties our observed ratio for this protostar agrees with the earlier ratios for Oort cloud comets. Comparing these different ratios for water directly assumes that the reservoirs, i.e., comets, Earth (planet) and inner protostellar region are linked and can be related.

The difference with the earlier estimates comes from the fact that those data are sensitive to much larger scales than the highresolution interferometric observations presented here, which directly image the water emission on 25-280 AU scales. Our interferometric observations provide a strong, model independent constraint on the deuteration of water in the innermost regions of protostars. That the low $\mathrm{HDO} / \mathrm{H}_{2} \mathrm{O}$ ratio in the warm gas is not much different from the cometary values is an indication that significant processing of the water between these early stages and the emerging solar system is not required.

Further high-resolution interferometric measurements toward larger samples of protostars will reveal whether the warm $\mathrm{HDO} / \mathrm{H}_{2} \mathrm{O}$ ratio is similar in different protostars. In particular, future high angular resolution observations with ALMA will be able to resolve possible variations in the $\mathrm{HDO} / \mathrm{H}_{2} \mathrm{O}$ ratio with distance from the central protostar and thereby show whether the slightly different ratios measured in different types of comets potentially could be related to their spatial origin in the protostellar envelope (Robert et al. 2000).
Acknowledgements. We thank the referee, Al Wootten, for insightful comments. The research at Centre for Star and Planet Formation is supported by the Danish National Research Foundation and the University of Copenhagen's programme of excellence. This research was furthermore supported by a Junior Group Leader Fellowship from the Lundbeck Foundation to JKJ. E.v.D. acknowledges the Netherlands Organization for Scientific Research (NWO) grant 614.001.008 and EU FP7 grant 291141 CHEMPLAN. This paper makes use of the following ALMA data: ADS/JAO.ALMA\#2011.0.00007.SV. The authors are grateful to JAO CSV team for planning and executing the ALMA data, and to Tim van Kempen and Markus Schmalzl of the Allegro node for help with data reduction. ALMA is a partnership of ESO (representing its member states), NSF (USA) and NINS (Japan), together with NRC (Canada) and NSC and ASIAA (Taiwan), in cooperation with the Republic of Chile. The Joint ALMA Observatory is operated by ESO, AUI/NRAO and NAOJ. The Submillimeter Array is a joint project between the Smithsonian Astrophysical Observatory and the Academia Sinica Institute of Astronomy and Astrophysics and is funded by the Smithsonian Institution and the Academia Sinica.

\section{References}

Alves, F. O., Vlemmings, W. H. T., Girart, J. M., \& Torrelles, J. M. 2012, A\&A, 542, A14

Bisschop, S. E., Jørgensen, J. K., Bourke, T. L., Bottinelli, S., \& van Dishoeck, E. F. 2008, A\&A, 488, 959

Blake, G. A., van Dishoeck, E. F., Jansen, D. J., Groesbeck, T. D., \& Mundy, L. G. 1994, ApJ, 428, 680

Bockelée-Morvan, D., Biver, N., Swinyard, B., et al. 2012, A\&A, 544, L15

Bottinelli, S., Ceccarelli, C., Neri, R., et al. 2004, ApJ, 617, L69

Caux, E., Kahane, C., Castets, A., et al. 2011, A\&A, 532, A23

Cazaux, S., Tielens, A. G. G. M., Ceccarelli, C., et al. 2003, ApJ, 593, L51

Chandler, C. J., Brogan, C. L., Shirley, Y. L., \& Loinard, L. 2005, ApJ, 632, 371

Chiang, H.-F., Looney, L. W., \& Tobin, J. J. 2012, ApJ, 756, 168

Coutens, A., Vastel, C., Caux, E., et al. 2012, A\&A, 539, A132

Geiss, J., \& Gloeckler, G. 1998, Space Sci. Rev., 84, 239

Hartogh, P., Lis, D. C., Bockelée-Morvan, D., et al. 2011, Nature, 478, 218

Jørgensen, J. K., \& van Dishoeck, E. F. 2010, ApJ, 710, L72

Jørgensen, J. K., Bourke, T. L., Myers, P. C., et al. 2005, ApJ, 632, 973

Jørgensen, J. K., Bourke, T. L., Nguyen Luong, Q., \& Takakuwa, S. 2011, A\&A, 534, A100

Jørgensen, J. K., Favre, C., Bisschop, S. E., et al. 2012, ApJ, 757, L4

Knude, J., \& Høg, E. 1998, A\&A, 338, 897

Kuan, Y.-J., Huang, H.-C., Charnley, S. B., et al. 2004, ApJ, 616, L27

Lécuyer, C., Gillet, P., \& Robert, F. 1998, Chem. Geol., 145, 249

Linsky, J. L. 2003, Space Sci. Rev., 106, 49

Liu, F.-C., Parise, B., Kristensen, L., et al. 2011, A\&A, 527, A19

Loinard, L., Torres, R. M., Mioduszewski, A. J., \& Rodríguez, L. F. 2008, ApJ, 675, L29

Loinard, L., Zapata, L. A., Rodriguez, L. F., et al. 2012, MNRAS, in press [arXiv: 1211.4744$]$

McMullin, J. P., Waters, B., Schiebel, D., Young, W., \& Golap, K. 2007, in Astronomical Data Analysis Software and Systems XVI, eds. R. A. Shaw, F. Hill, \& D. J. Bell, ASP Conf. Ser., 376, 127

Müller, H. S. P., Thorwirth, S., Roth, D. A., \& Winnewisser, G. 2001, A\&A, 370 , L49

Parise, B., Simon, T., Caux, E., et al. 2003, A\&A, 410, 897

Parise, B., Caux, E., Castets, A., et al. 2005, A\&A, 431, 547

Persson, M. V., Jørgensen, J. K., \& van Dishoeck, E. F. 2012, A\&A, 541, A39

Pickett, H. M., Poynter, R. L., Cohen, E. A., et al. 1998, J. Quant. Spectrosc. Radiat. Trans., 60, 883

Pineda, J. E., Maury, A. J., Fuller, G. A., et al. 2012, A\&A, 544, L7

Qi, C. 2008, The MIR Cookbook, The Submillimeter Array, Harvard-Smithsonian Center http: //cfa-www.harvard. edu/ cqi/mircook.html

Robert, F., Gautier, D., \& Dubrulle, B. 2000, Space Sci. Rev., 92, 201

Sault, R. J., Teuben, P. J., \& Wright, M. C. H. 1995, in Astronomical Data Analysis Software and Systems IV, eds. R. A. Shaw, H. E. Payne, \& J. J. E. Hayes, ASP Conf. Ser., 77, 433

Stark, R., Sandell, G., Beck, S. C., et al. 2004, ApJ, 608, 341

van Dishoeck, E. F., Blake, G. A., Jansen, D. J., \& Groesbeck, T. D. 1995, ApJ, 447,760

Villanueva, G. L., Mumma, M. J., Bonev, B. P., et al. 2009, ApJ, 690, L5

Wilson, T. L., \& Rood, R. 1994, ARA\&A, 32, 191

Page 5 is available in the electronic edition of the journal at http://www . aanda.org 
M. V. Persson et al.: Warm water deuterium fractionation in IRAS 16293-2422

\section{Appendix A}

Table A.1. Relevant parameters from the molecular line catalogs for the observed lines.

\begin{tabular}{lllllllll}
\hline \hline $\begin{array}{l}\text { Line } \\
\mathrm{Id}^{a}\end{array}$ & Species & $\begin{array}{l}\text { Frequency } \\
{[\mathrm{GHz}]}\end{array}$ & Transition & $\begin{array}{l}\text { Line strength } \\
{\left[\text { Debye }^{2}\right]}\end{array}$ & $\begin{array}{l}E_{\mathrm{u}} \\
{[\mathrm{K}]}\end{array}$ & $\begin{array}{l}\text { Beam } \\
\text { Size }(\mathrm{PA})\end{array}$ & $\begin{array}{l}\text { Resolution } \\
{\left[\mathrm{km} \mathrm{s}^{-1}\right]}\end{array}$ & $\begin{array}{l}\mathrm{rms} \\
{\left[{ }^{b}\right]}\end{array}$ \\
\hline 1 & $\mathrm{H}_{2}^{18} \mathrm{O}$ & 203.40752 & $3_{1,3}-2_{2,0}$ & 0.34 & 203.7 & $3.1^{\prime \prime} \times 5.0^{\prime \prime}\left(-25.3^{\circ}\right)$ & 0.30 & 208 \\
2 & $\mathrm{HDO}$ & 225.89672 & $3_{1,2}-2_{2,1}$ & 0.69 & 167.6 & $2.3^{\prime \prime} \times 3.9^{\prime \prime}\left(15.0^{\circ}\right)$ & 0.54 & 190 \\
3 & $\mathrm{H}_{2}^{18} \mathrm{O}$ & 692.07914 & $5_{3,2}-4_{4,1}$ & 1.26 & 727.6 & $0^{\prime} 17 \times 0^{\prime \prime} 29\left(109.8^{\circ}\right)$ & 0.40 & 82 \\
\hline
\end{tabular}

Notes. ${ }^{(a)}$ Identifies the lines in Table $1 .{ }^{(b)} \mathrm{mJy} \mathrm{beam}^{-1}$ channel $^{-1}$. 